$$
\begin{aligned}
& 1 w-37 \\
& 175558
\end{aligned}
$$

NASA Technical Memorandum 106253

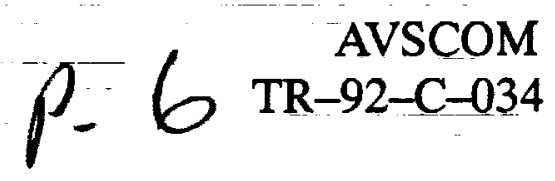

\title{
Efficient Fault Diagnosis of Helicopter Gearboxes
}

H. Chin and K. Danai

University of Massachusetts

Amherst, Massachusetts

and

D.G. Lewicki

Propulsion Directorate

U.S. Army Aviation Systems Command

Lewis Research Center.

Cleveland, Ohio

Prepared for the
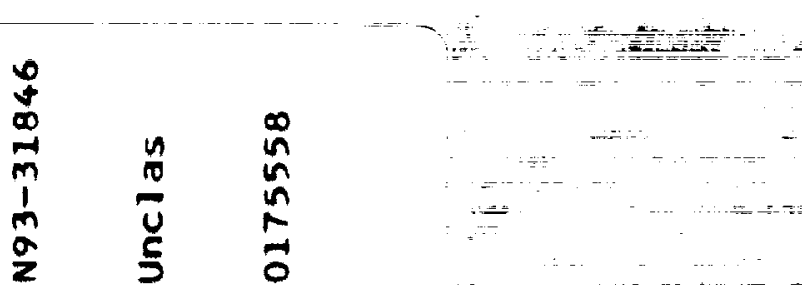

$\sum_{\hat{o}}^{n}$

$E$

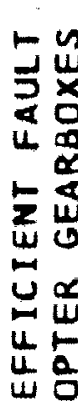

natis
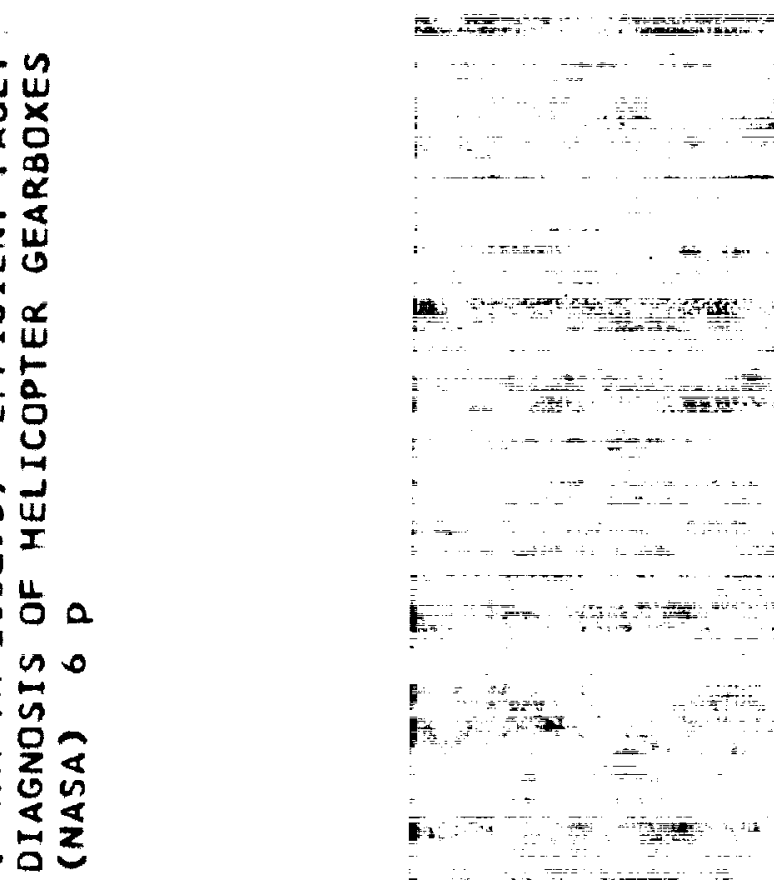

12th World Congress International Federation of Automatic Control

Sydney, Australia, July 19-23, 1993 


\title{
EFFICIENT FAULT DIAGNOSIS OF HELICOPTER GEARBOXES
}

\author{
H. Chin*, K. Danai*, and D. G. Lewicki** \\ *Department of Mechanical Engineering, University of Massachusetts, Amherst, MA 01009, USA \\ * U.S. Army Research Laboratory, Vehicle Propulsion Directorate, NASA Lewis Research Center, Cleveland, \\ $O B$ \$4195, USA
}

\begin{abstract}
Abst ract. Application of a diagnostic system to a helicopter gearbax is presented. The diagnostic system is a nonparametric pattern classifier that uses a multi-valued infiuence matrix (MVIM) as its diagnostic model and benefits from a fast learning algorithm that enables it to estimate its diagnostic model from a small number of measurement-fauit data. To test this diagnostic system, vibration measurements were collected from a helicopter gearbox test stand during accelerated fatigue tests and at various fault instances. The diagnostic results indicate that the MVIM system can accurately detect and diagnose various gearbox faults so long as they are inciuded in training.
\end{abstract}

\section{INTRODUCTION}

Helicopter drive trains are significant contributors to both maintenance cost and tlight safety incidents. Drive trains comprise almost $30 \%$ of maintenance costs and $16 \%$ of mechanically related malfunctions that often result in the loss of aircraft (Chin, 1993). As such, it is crucial that faults be diagnosed in-fight so as to prevent loss of lives.

Fault diagnosis of helicopter power trains is based primarily on vibration monitoring. As such, considerable effort has been directed toward the identification of features of vibration that are affected by specific faults (e.g., Pratt, 1986), and the development of signal processing techniques that can quantify such features. The main problem with this approach, however, is that due to the complexity of belicopter gearboxes and the interaction between their various components, the individual vibration features do not provide a reliable basis for diagnosis.

As an alternative to single-feature based diagnosis, fault diagnosis based on several features can be performed using pattern classification ( $\mathrm{Pau}, 1981$ ). Among the various pattern classifiers used for diagnosis, artificial neural nets are the most notable due to their nonparametric nature (independence of the probabilistic structure of the system) and their ability to generate complex decision regions. However, neural nets generally require extensive training to develop the decision regions (diagnostic model). In cases such as helicopter power trains, where adequate data may not be available for training, artificial neural nets may misdiagnose the fault.

In this paper we demonstrate the application of a diagnostic method that can establish the fault signatures based on a small number of measurement-fault data. This method utilizes a multi-valued influence matrix (MVIM) as its diagnostic model which provides indices for diagnosability of the system and variability of the fault signatures (Danai and Chin, 1991). These indices are used as feedback to improve fault sigmatures through adaptation (Chin and Danaj. 1993).

\section{THE MVIM METHOD}

Measurements are processed in the MVIM method as illustrated in Fig. 1. They are usually filtered first to obtain a vector of processed measurements $P$, then they are converted to binary numbers through a flagging operation (i.e., abnormal measurements characterized by ' 1 ' and normal ones by ' 0 '), and finally they are analyzed through the diagnostic model. In the MVIM method, flagging is performed by a Flagging Unit that is tuned according to measures of diagnosability and fault signature variability obtained from MVM so as to improve the fault signatures. The MVIM method is explained in detail in (Danai and Chin, 1991) and (Chin and Danai, 1992). We will only discuss the overall concept here for completeness.

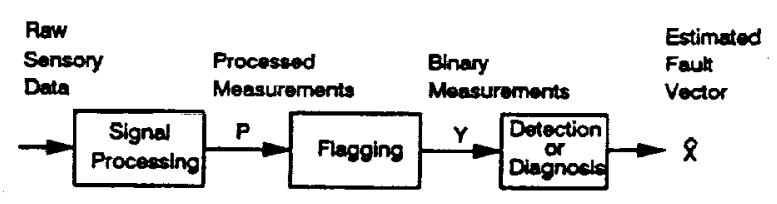

Fig. 1: Processing of measurements in the MVIM method

\subsection{Fault Signature Representation}

Fault signatures in the MVIM method are represented by the $n$ unit-length columns $\overline{\mathrm{V}}_{j} \in \mathcal{R}^{n}$ of a multi-valued influence matrix (MVIM) $\bar{A}$ :

$$
\overline{\mathbf{A}}=\left[\begin{array}{lllll}
\overline{\mathbf{V}}_{1}, & . ., & \overline{\mathbf{V}}_{j}, \ldots, & \overline{\mathbf{V}}_{n}
\end{array}\right]
$$

where $m$ denotes the number of measurements, and $n$ represents the number of faults. Based upon this influence matrix, the faults can be ranked according to their possibility of occurrence by the closeness of their influence vectors $\bar{V}_{j}$ to the vector of flagged measurements $Y$ (see Fig. 2). In the MVIM method, the vector of diagnostic certainty measures, $\hat{\mathrm{X}}$, which ranks the faults according to 
their possibility of occurrence is defined as

$$
\hat{\mathbf{X}}=\left\{\hat{x}_{1}, \ldots, \hat{x}_{j}, \ldots, \hat{x}_{n}\right\}^{T}=\cos \left\{\alpha_{1}, \ldots, \alpha_{j}, \ldots, \alpha_{n}\right\}^{T}
$$

where the $\hat{x}_{j}$ represent the individual diagnostic certainty mensures, and the $\alpha_{j}$ denote the individual angles between the influence vectors $\bar{V}_{j}$ and the flagged measurement vector $Y$ (see Fig. 2).

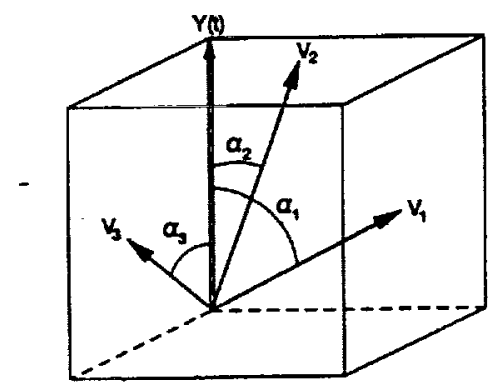

Fig. 2: Schematic of diagnostic reasoning in the MVIM method, illustrated in three dimensional space

\subsection{Estimation of $\overline{\mathbf{A}}$}

The influence vectors in Eq. (1) are not known a-priori and need to be estimated. In the MVIM method, the error in diagnosis is used as the basis to estimate/update the influence vectors. For this purpose, the fault signatures are updated recursively after the occurrence of each fault to minimize the sum of the squared diagnostic error associated with that fault (Danai and Chin, 1991).

\subsection{Fault Signature Evaluation}

One of the unique features of the MVIM method is its ability to evaluate quantitatively the uniqueness and variability of fault signatures, so that these quantitative measures can be used to improve the flagging operation. In the MVIM method, the uniqueness of fault signatures is represented by the closeness of pairs of influence vectors, and the index of diagnosability is defined to characterize the closest pair of fault signatures.

In the MVIM method, the variability of fault signatures is defined by their variance. For this purpose, the variance matrix of $\bar{A}$ is estimated to provide a measure of the variations of individual components of $\bar{A}$. Since in the MVIM method the components of $\overline{\mathbf{A}}$ are adjusted recursively, the variance matrix can be readily estimated during training (Chin and Danai, 1993). The index of fault signature variability is defined as the largest component of the variance matrix, representing the largest variability in the components of $\bar{A}$.

\subsection{Flagging}

The influence matrix $\overline{\mathbf{A}}$ is estimated based on the values of the flagged measurement vector $Y$. Thus, before the influence matrix is used for diagnostic reasoning, the integrity of the flagging operation needs to be ensured. Flagging in the MVIM method is performed by a Flagging Unit that is tuned to improve the diagnosability of the system and reduce the variability of the fault signatures (Chin and Danai, 1993).

The Flagging Unit uses a sample set of measurement-fault vectors to tune its parameters iteratively. After each pass through the training batch, the Flagging Unit counts the total number of false alarms and undetected faults, and estimates the uniqueness and variability of the estimated fault signatures from the current values of the influence matrix, so that it can use these measures as feedback in the next adaptation round. Adaptation stops when the total number of false alarms and undetected faults are minimized, and the uniqueness and consistency of fault signatures are enhanced.

The Flagging Unit processes the residuals as follows (see Fig. 3). The residual vector $P \in \mathcal{R}^{m}$ is first passed through Hard-Limiter I (consisting of a vector of $m$ thresholds, $h_{1 i}$, $i=1, \ldots, m)$ to produce a binary vector $Z \in B^{m}$. This vector is then multiplied sequentially by the normalized columns of a Quantization Matrix and then thresholded by Hard-Limiter II to produce the individual components of the flagged measurement vector $Y \in B^{m}$. Training of the Flagging Unit comprises of adjusting the thresholds of Hard-Limiters I and II and the Quantization Matrix, as explained in detail in (Chin and Danai, 1992).

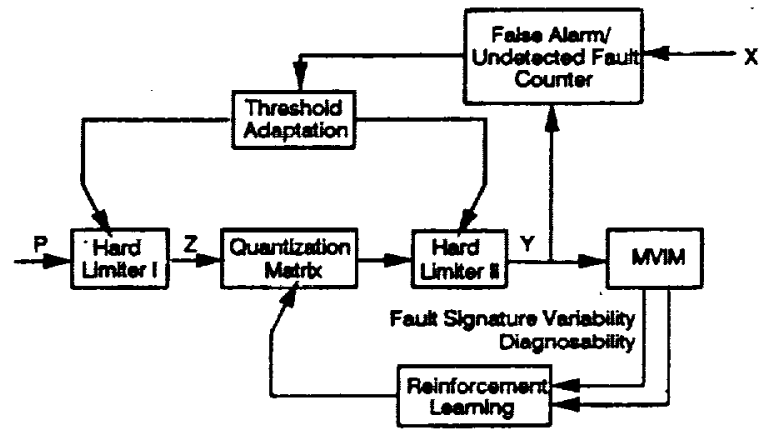

Fig. 3: Schematic of the Flagging Unit

\section{EXPERIMENTAL}

Vibration data were collected at NASA Lewis Research Center. Various component failures in an OH-58A main rotor transmission were produced during the experiments (Lewicki et al., 1992). The configuration of the transmission which was tested in the NASA 500-hp Helicopter Transmission Test Stand is shown in Fig. 4. The vibration signals were recorded from eight piezoelectric accelerometers (frequency range of up to $10 \mathrm{KHz}$ ) using an FM tape recorder. The signals were recorded once every bour for about one to two minutes per recording (at the tape speed of $30 \mathrm{in} / \mathrm{sec}$, providing a bandwidth of $20 \mathrm{KHz}$ ).

A total of five tests were performed, where each test was rin between nine to fifteen days for approximately four to eight hours a day. New components were used at the start of each test. When a component fault was detected during a test, it was replaced with a new one for the remainder of the test. Eleven failures occurred during these tests. The status of various faults during the five tests are shown in Table 1 where no-fault cases are denoted by $x_{0}$. In Test \#1, spiral bevel pinion failure $\left(x_{4}\right)$ is estimated to have been present on days 5-9 with sun gear failure $\left(x_{1}\right)$ also occurring on day 9 . No failures occurred in Test \#2, so all the nine days for this test are marked as normal. In Test \#3, planet bearing failure $\left(x_{2}\right)$ was established to have been present on days 3-4 and 11-12, with housing crack $\left(x_{3}\right)$ and mast bearing failure $\left(x_{6}\right)$ occurring on day 9 and day 13 , respectively. Test \#4 is estimated to have contained planet bearing failure on days 11-12 and sun gear failure on days 14-15. In Test \#5, housing crack 


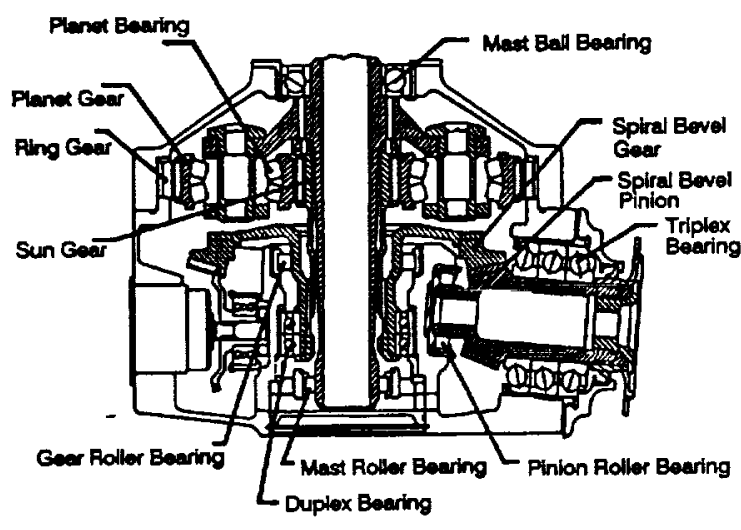

Fig. 4: Configuration of the OH-58A main rotor transmission

is assumed to have been present on days $7-11$, with sun gear failure $\left(x_{1}\right)$ and planet gear failure $\left(x_{5}\right)$ occurring on days 10-11 and day 11 , respectively.

Table 1: Association of data from each day of the five tests with the no-fault case $x_{0}$ and various fault cases. The siz faults are represented as $x_{1}$ : sun gear failure, $x_{2}$ : planet bearing failure, $x_{3}$ : housing crack, $x_{4}$ : spiral bevel pinion failure, $x_{5}$ : planet gear failure, and $x_{6}$ : mast bearing failure

\begin{tabular}{cccccc}
\hline & \multicolumn{5}{c}{ Fault Status } \\
\cline { 2 - 6 } Day & Test \#1 & Test \#2 & Test \#3 & Test \#4 & Test \#5 \\
\hline 1 & $x_{0}$ & $x_{0}$ & $x_{0}$ & $x_{0}$ & $x_{0}$ \\
2 & $x_{0}$ & $x_{0}$ & $x_{0}$ & $x_{0}$ & $x_{0}$ \\
3 & $x_{0}$ & $x_{0}$ & $x_{2}$ & $x_{0}$ & $x_{0}$ \\
4 & $x_{0}$ & $x_{0}$ & $x_{2}$ & $x_{0}$ & $x_{0}$ \\
5 & $x_{4}$ & $x_{0}$ & $x_{0}$ & $x_{0}$ & $x_{0}$ \\
6 & $x_{4}$ & $x_{0}$ & $x_{0}$ & $x_{0}$ & $x_{0}$ \\
7 & $x_{4}$ & $x_{0}$ & $x_{0}$ & $x_{0}$ & $x_{3}$ \\
8 & $x_{4}$ & $x_{0}$ & $x_{0}$ & $x_{0}$ & $x_{3}$ \\
9 & $x_{4}, x_{1}$ & $x_{0}$ & $x_{3}$ & $x_{0}$ & $x_{3}$ \\
10 & & & $x_{0}$ & $x_{0}$ & $x_{3}, x_{1}$ \\
11 & & & $x_{2}$ & $x_{2}$ & $x_{3}, x_{1}, x_{5}$ \\
12 & & & $x_{2}$ & $x_{2}$ & \\
13 & & & $x_{6}$ & $x_{0}$ & \\
14 & & & & $x_{1}$ & \\
15 & & & & $x_{1}$ & \\
\hline
\end{tabular}

\section{SIGNAL PROCESSING}

In order to identify the effect of faults on the vibration data, the vibration signals obtained from the five tests were digitized and processed by a commercially available signal analyzer (Stewart Hughes, 1987) with four processing modules: (1) Statistical Analysis (STAT), (2) Basebend Power Spectrum Analysis (BBPS), (3) Bearing Analysis (BRGA), and (4) Signal Averaging Analysis (SGAV).

For analysis purposes, only one data record per day was used for each test. The data records were taken at the beginning of the day unless a fault was reported. When a fault was detected, the secord was taken right before the fault incident to ensure that the data record reflected the fault. Also, in order to reduce estimation errors, each data record was partitioned into sixteen segments, and param- eters were estimated for each segment and averaged over these segments. The data records as well as the parameters obtained from the above processing modules were then transferred to a personal computer for further analysis (Chin, 1992).

\section{IMPLEMENTATION AND RESULTS}

The configuration of the MVIM system as applied to fault diagnosis of the OH-58A main rotor transmission is illustrated in Fig. 5. As shown in this figure, two MVIMs are trained for each accelerometer. One MVIM to perform detection (i.e., to determine whether a fault has occurred or not), and a diagnostic MVIM to isolate the fault. The 54 parameters obtained from the signal analyzer were used to train and test the MVIM system. The detection MVIM contained only two columns to characterize the nofault and fault signatures, whereas the diagnostic MVM contained seven columns, one characterizing the no-fault signature and the other six representing the signatures of individual faults. Note that the two MVIMs can be percejved as filters with different resolutions. In order to integrate the results from the MVIMs associated with the eight accelerometers, a voting scheme was utilized. Test

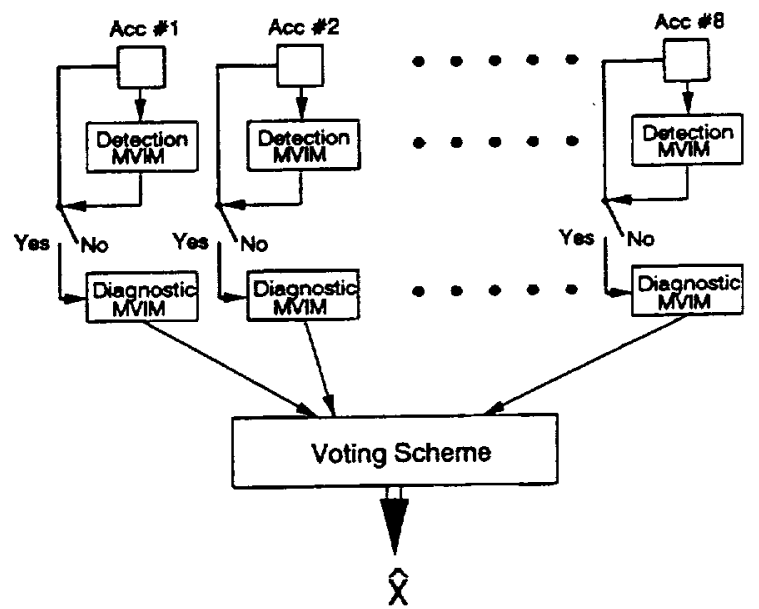

Fig. 5: Configuration of the MVIM system as applied to the OH-58A main rotor transmission.

\#3 and \#4 contained most of the failure modes (i.e., 4 out of 6 ). Therefore, the parameters from these two tests were used to train the MVIMs. Note that not all of the failure modes were included in training, so the test results were not expected to be perfect. For training the detection MVIMs, only the 19 parameters from the $S T A T, B B P S$, and $B R G A$ modules were used. Previous studies on this data show that these 19 parameters are adequate for detection (Chin, 1993). For training the diagnostic MVIMs, all of the 54 parameters were utilized.

The initial values of the detection MVIMs $(19 \times 2)$ and diagnostic MVIMs $(54 \times 7)$ were each set to $O$ (i.e., matrices with all zero entries), and the initial values of the Quantization Matrices ( $19 \times 19$ for detection) and ( $54 \times 54$ for diagnosis) were set to jdentity matrices. The initial threshold levels for Hard-Limiter I were set at the mean plus one standard deviation of the corresponding parameter, and for Hard-Limiter II, they were set at 0.5 . The maximum number of epochs for training the detection and diagnostic MVIMs was set to 50 . After each epoch, the detection/diagnostic performance of MVIMs within the training 
set was tested. Training was stopped once perfect detection/diagnosis was achieved, to avoid overtraining (Hertz et al, 1991). The number of epochs used for individual detection MVMMs were: $8,5,50,37,50,15,50$, and 50 for accelerometers \#1 to \#8, respectively, whereas for diagnostic MVTMs they were: $50,1,2,2,26,50,50$, and 50. According to the number of epochs used for individual MVMMs, it is clear that the detection MVIMs associated with accelerometers \#3, \#5, \#7, and \#8 did not achieve perfect detection within the training set. Similarly, the diagnostic MVIMs associated with accelerometers \#1, \#6, \#7, and \#8 did not achieve perfect diagnosis within the training set.

The MVins trained on Tests \#3 and \#4 were evaluated for all of the tests. For this purpose, the 19 parameters from each of the eight accelerometers were first passed through the corresponding detection MVIM for all of the five tests to reflect the occurrence of faults. Once a fault was posted by a detection MVIM, the set of 54 parameters from that accelerometer was passed through the corresponding diagnostic MVIM. At the final stage, the diagnostic certainty measures obtained from the eight diagnostic MVIMs were consolidated by the voting scheme, which utilized weights reflecting the speed of convergence of individual fault signatures during training (Chin, 1993). As such those influence vectors which converged faster were assigned larger weights and vice versa. Zero weights were assigned to the influence vectors which did not converge during training, and unity weights to those which converged within one epoch.

The diagnostic results obtained from the above diagnostic system are shown in Table 2. The results indicate that the MVIM system was able to produce perfect diagnostics for Tests \#3 and \#4, on which it was trained, and that it diagnosed $88 \%$ of the faults in all of the tests. Specifically, the results in Table 2 indicate that the MVIM system produced two false alarms (on day 4 of Test \#1 and day 6 of Test \#5), and five misdiagnoses (on days 5-8 of Test \#1 and day 11 of Test \#5). In addition, this system produced equal diagnostic certainty measures for the no-fault case and sun gear failure on day 10 of Test \#5 and could only diagnose one of the faults on day 9 of Test \#1, and on days 10 and 11 of Test \#5. However, it should be noted that faults $x_{4}$ and $x_{5}$ were not included in training, so no fault signatures were estimated for them. The correct diagnostic rate of MVIM, with these two faults excluded would be over $95 \%$, which is quite noteworthy considering that the MVIM system was trained on a small number of measurement-fault data with very few repetitions of each fault.

\section{CONCLUSION}

A fault diagnostic system based on the MVTM method is applied to a helicopter gearbox for which only a small number of measurement-fault data was available. This diagnostic system utilizes two levels of isolation and integrates the results obtained from various accelerometers through a voting scheme. The diagnostic results indicate that the MVIM system correctly detected all of the fault incidents, with only two false alarms posted. The results further indicate that this system correctly diagnosed all of the faults it was trained for.
Table 2: Estimated fault status from each day of the five tests. Same notations are adopted as in Table 1

\begin{tabular}{cccccc}
\hline & \multicolumn{5}{c}{ Estimated Fault Status } \\
\cline { 2 - 6 } Day & Test \#1 & Test \#2 & Test \#3 & Test \#4 & Test \#5 \\
\hline 1 & $x_{0}$ & $x_{0}$ & $x_{0}$ & $x_{0}$ & $x_{0}$ \\
2 & $x_{0}$ & $x_{0}$ & $x_{0}$ & $x_{0}$ & $x_{0}$ \\
3 & $x_{0}$ & $x_{0}$ & $x_{2}$ & $x_{0}$ & $x_{0}$ \\
4 & $x_{3}$ & $x_{0}$ & $x_{2}$ & $x_{0}$ & $x_{0}$ \\
5 & $x_{3}$ & $x_{0}$ & $x_{0}$ & $x_{0}$ & $x_{0}$ \\
6 & $x_{3}$ & $x_{0}$ & $x_{0}$ & $x_{0}$ & $x_{6}$ \\
7 & $x_{3}$ & $x_{0}$ & $x_{0}$ & $x_{0}$ & $x_{3}$ \\
8 & $x_{3}$ & $x_{0}$ & $x_{0}$ & $x_{0}$ & $x_{3}$ \\
9 & $x_{1}$ & $x_{0}$ & $x_{3}$ & $x_{0}$ & $x_{3}$ \\
10 & & & $x_{0}$ & $x_{0}$ & $x_{0}, x_{1}$ \\
11 & & & $x_{2}$ & $x_{2}$ & $x_{2}, x_{6}$ \\
12 & & & $x_{2}$ & $x_{2}$ & \\
13 & & & $x_{6}$ & $x_{0}$ & \\
14 & & & & $x_{1}$ & \\
15 & & & & & $x_{1}$
\end{tabular}

\section{ACKNOWLEDGEMENTS}

The authors would like to express their gratitude to Sikorsky Aircraft Company for its continued support of this project and NASA for providing the experimental data. This work was supported in part by the National Science Foundation (Grants No. DDM-9015644 and MSS9102149).

\section{REFERENCES}

Chin, H. (1992). Vibration Analysis of an OH-58A Main Rotor Transmission, Technical Report, Department of Mechanical Engineering, University of Massachusetts, Amherst, MA 01003.

Chin, H. (1993). A Non-parametric Pattern Classifying Diagnostic Method and Its Application, Ph.D. Dissertation, Department of Mechanical Engineering, University of Massachusetts, Amberst, MA 01003.

Chin, H., and K. Danai (1993). Improved Flagging for Pattern Classifying Diagnostic Systems, IEEE Trans. on Systems, Man, and Cybernetics, in press.

Danai, K., and H. Chin (1991). Fault Diagnosis with Process Uncertainty, ASME J. of Dynamic Systems, Measurement, and Control, Vol. 113, No. 3, pp. 339 -343 .

Hertz, J., A. Krogh, and R. G. Palmer (1991). Introduction to the Theory of Neural Computation, AddisonWesley, Redwood City, CA.

Lewicki, D. G., H. J. Decker, and J. T. Shimski (1992). Full-Scale Transmission Testing to Evaluate Advanced Lubricants, Technical Report, NASA TM105668, AVSCOM TR-91-C-035, NASA Lewis Research Center, Cleveland, OH 44135.

Pau, L. F. (1981). Failure Diggnosis and Performance Monitoring, Marcel Dekker, New York, NY.

Pratt, J. L. (1986). Engine and Transmission Monitoring - A Summary of Promising Approaches, Mechanical Failure Prevention Group 41th Meeting, Oct., pp. 229-236.

Stewart Hughes (1987). Transmission Systems Analysis for the MSDA, MMS5: 2nd edition, Stewert Hughes Limited, Southhampton, U. K. 
Public reporting burden for this collection of information is estimated to average 1 hour per response, including the time for reviewting instructions, searching existing data sources, pubre gathocion Dovis Hion of inay, Suite 1204, Arlington, VA 22202-4302, and to the Ottice of Management and Budget, Paperwork Reduction Project (0704-018B). Washington, DC 20503.

\begin{tabular}{|l|c|c|}
\hline 1. AGENCY USE ONLY (Leave blank) & $\begin{array}{r}\text { 2. REPORT DATE } \\
\text { July } 1993\end{array}$ & $\begin{array}{r}\text { 3. REPORT TYPE AND DATES COVERED } \\
\text { Technical Memorandum }\end{array}$ \\
\hline
\end{tabular}

4. TITLE AND SUBTTILE

Efficient Fault Diagnosis of Helicopter Gearboxes

6. AUTHOA(S)

H. Chin, K. Danai, and D.G. Lewicki

7. PEAFORMING ORGANZATION NAME(S) AND ADDRESS(ES)

NASA Lewis Research Center

Cleveland, Ohio 44135-3191

and

Propulsion Directorate

U.S. Army Aviation Systems Command

Cleveland, Ohio 44135-3191

9. SPONSORING/MONTORING AGENCY NAME(S) AND ADDRESS(ES)

National Aeronautics and Space Administration

Washington, D.C. 20546-0001

and

U.S. Army Aviation Systems Command

Cleveland, Ohio 44135-3191
WU-505-62-10

1L162211A47A

11. SUPPLEMENTARY NOTES

Prepared for the 12th World Congress International Federation of Automatic Control, Sydney, Australia, July 19-23, 1993. H. Chin and K. Danai, University of Massachusetts, Department of Mechanical Engineering, Amherst, Massachusetts 01003, and D.G. Lewicki, Propulsion Directorate, U.S. Amy Aviation Systems Command. Responsible person, D.G. Lewicki, (216) 433-3970.

12a. DISTRIBUTION/AYAILABIUTY STATEIENT

12b. DISTRIBUTION CODE

Unclassified - Unlimited

Subject Category 37

13. ABSTRACT (Maximum 200 words)

Application of a diagnostic system to a helicopter gearbox is presented. The diagnostic system is a nonparametric pattem classifier that uses a multi-valued influence matrix (MVIM) as its diagnostic model and benefits from a fast leaming algorithm that enables it to estimate its diagnostic model from a small number of measurement-fault data. To test this diagnostic system, vibration measurements were collected from a helicopter gearbox test stand during accelerated fatigue tests and at various fault instances. The diagnostic results indicate that the MVIM system can accurately detect and diagnose various gearbox faults so long as they are included in training.

14. SUBJECT TERMS

Failure detection; Monitoring; Pattern recognition

15. NUMBER OF PAGES

6

16. PRICE CODE

$\mathrm{A} 02$

17. SECUATY CLASSIFICATION OF REPORT

Unclassified
18. SECURTY CLASSIFICATION OF THIS PAGE

Unclassified
19. SECURTY CLASSIFICATION OF ABSTRACT

Unclassified 


.

